\title{
Deep Learning Methods for Autism Spectrum Disorder Diagnosis Based on fMRI Images
}

\author{
(D) Muhammed Ali Bayram¹, (Dilyas Özer ${ }^{2}$, (D) Feyzullah Temurtaş3, \\ ${ }^{1}$ Corresponding Author; Electrical-Electronics Engineering Department, Bandırma Onyedi Eylül University; \\ muhammedbayram@ogr.bandirma.edu.tr \\ ${ }^{2}$ Computer Engineering Department, Bandırma Onyedi Eylül University; \\ iozer@bandirma.edu.tr \\ ${ }^{3}$ Electrical-Electronics Engineering Department, Bandırma Onyedi Eylül University; \\ ftemurtas@bandirma.edu.tr
}

Received 13 February 2021; Revised 06 March 2021; Accepted 23 March 2021; Published online 02 April 2021

\begin{abstract}
Brain injuries are significant disorders affecting human life. Some of these damages can be completely eliminated by methods such as drug therapy. On the other hand, there is no known permanent treatment for damages caused by diseases such as Alzheimer, Autism spectrum disorder (ASD), Multiple sclerosis and Parkinson. Treatments aimed at slowing the progression of the disease are generally applied in these types of disorders. For this reason, essential to diagnose the disease at an early phase before behavioral disorders occur. In this study, a study is presented to detect ASD through resting-state functional magnetic resonance imaging rs-fMRI. However, fMRI data are highly complex data. Within the study's scope, ASD and healthy individuals were distinguished on 871 samples obtained from the ABIDE I data set. The long short-term memory network (LSTM), convolutional neural network (CNN), and hybrid models are used together for the classification process. The results obtained are promising for the detection of ASD on fMRI.
\end{abstract}

Keywords: Deep learning, RNN, rs-fMRI, Autism Spectrum Disorder

\section{Introduction}

The brain is the core member of the nervous system and one of the human body's essential organs [1]. For this reason, the functioning of the brain has always been an area of interest for researchers. Brain functions may be partially or wholly impaired over time for many reasons, such as living conditions and genetic factors. These impairments in brain functions lead to the emergence of some diseases. Multiple sclerosis (MS), Attention deficit hyperactivity disorder (ADHD), Parkinson's, ASD and depression are some of these diseases. Depression, one of the disorders, can be treated entirely with drugs [1].

On the other hand, there is no known definitive treatment for diseases such as MS, Parkinson's, and ASD [1]. Therefore, the progression of the disease is controlled rather than curing it completely. However, in most cases, diseases can only be diagnosed after complications have occurred. In this situation, patients are exposed to irreversible damage [1]. In this respect, diagnosing diseases at an early stage is one of the critical issues.

Many different methods are used to detect impairments in brain functions. Methods such as magnetoencephalography (MEG) and positron emission tomography (PET) are some of them. Similarly, Electroencephalography (EEG) and rs-fMRI are some of the methods used. Compared to other methods, rs-fMRI provides much better spatial resolution [2]. Besides, it provides a temporal resolution of brain functions [2]. In this noninvasive method, activities in the brain are evaluated by following the movements in oxygen levels in the blood [2,3]. Two different methods are used for fMRI [1]. These are called task-based fMRI and resting-state fMRI [1]. It is possible to diagnose ASD using the rs-fMRI method [4].

ASD is an extremely complicated neurodevelopmental disorder and it is getting more common day by day $[1,4]$. Many comorbid disorders such as seizures, anxiety, and intellectual impairment can occur in 
Bayram et al.

this disease [5,6]. According to a report prepared in 2013, it appears that one in 55 children between the ages of 6 and 17 is diagnosed with ASD [7]. Mild or severe disorders that affect social interaction, communication, behavior, and imagination can be seen in patients with ASD [8-11]. Until today, diagnoses of ASD in childhood have generally been made based on the data obtained from clinical interviews and behavioral observations [1]. One of the most significant weaknesses in traditional diagnostic techniques is that the diagnosis can be made after substantial behavioral disorders occur [1]. On the other hand, it is crucial to diagnose these disorders early before behavioral disorders arise [1]. It is vital to improving diagnostic accuracy based on medical imaging techniques such as fMRI $[12,13]$.

In recent years, studies on the use of machine learning (ML) techniques have gained momentum to increase the accuracy of diagnosis in neurological disorders and reduce physicians' workload [1,4]. ML are artificial intelligence applications that can automatically learn and detect patterns over a group of data $[14,15]$. ML has been used successfully in many tasks, where using traditional algorithms is difficult or not possible. One of the essential advantages of machine learning is making consistent and high-performance predictions using complex and nonlinear relationships between features [16]. In this direction, they can identify complex relationships that people cannot see directly. Accordingly, ML algorithms have been used successfully in different tasks such as disease diagnosis [17-20] and evaluation of EEG data [19,21].

With the Autism Brain Imaging Data Exchange I (ABIDE I) [22] data set, studies for detecting ASD on fMRI images gained speed [1]. In this direction, in the study conducted in [23], Various algorithms such as Linear Discriminant Analysis (LDA), Random Forest (RF) and Support Vector Machine (SVM) were used in the ABIDE data set. In addition, neural network algorithms such as Multi-Layer Perceptron (MLP) are also used for ASD detection in the ABIDE data set. ASD detection has been carried out. The tests performed achieved the highest performance by using MLP with 56.26\% [23]. In another study performed using a CNN on ABIDE I data set, a classification success of $72.73 \%$ was obtained [24]. In another study, only NYU Langone Medical Center data were used instead of the entire ABIDE I data set [4]. A success of $90.39 \%$ was achieved in the tests performed using the deep neural network. On the other hand, using data from only one clinic instead of the entire data set stands out as an essential deficiency. When the entire whole ABIDE set is evaluated together, it is seen that the data is obtained from different brand devices in different locations and under different settings. In this case, it stands out as a factor that significantly affects the classification accuracy.

In this study, hybrid approaches using CNN, LSTM and both are suggested for ASD detection on fMRI images. While CNNs are very successful in detecting spatial dependencies, LSTMs are very successful in detecting temporal dependencies. Accordingly, it is evaluated that hybrid models formed by CNN and LSTM models on fMRI data with temporal and spatial dependencies can provide more successful results by detecting both spatial and temporal dependencies. The results obtained are promising in terms of detecting ASD on medical images.

\section{Materials and Methods}

\subsection{Datasets}

Used the ABIDE 1 dataset for making our study comparable with existing studies [22]. The dataset includes rs-fMRI brain images, phenotypic information and structural MRI (T1-weighted) of 1112 subjects, composed of 2 different groups, 539 ASD and 573 typical controls (TC). The data are gathered from 17 various institutions and brought together and made available to all researchers for use in scientific research. Studying on the ABIDE 1 data set is difficult due to the heterogeneity of the subjects, large number of diversity, and the lack of some data sets within the dataset. ABIDE 1 dataset is accessible on various websites of organizations operating in this field. Since these websites belonging to independent communities from each other receive the data independently, the differences between the data on the websites are very critical. To make our study consistent, we used the same 871 samples from the ABIDE 1 data set [23], [24], [25]. Of the 871 subjects, 403 patients were ASD and 468 were TC patients. 
Bayram et al.

\subsection{Brain Network}

As is known, a network consists of edges and nodes. Creating a network representing a brain with functional MR data is quite complex, as it is difficult to detect intricate nodes and edges [26], [27]. If edges and nodes are misidentified, the network that studying can become extremely difficult to analyze [28]. The two most commonly used methods to describe a network are ROI-based [24] and voxel-based [29] parcellation schemes. In voxel-based approaches, each voxel in the MRI data is defined as a node. In addition, the connection between each voxel is expressed as an edge [30]. In this approach, ROIs are not considered to have the main effect on brain function [31]. Additionally, in ROI parcellation schemes, the data of the human brain is split into several different ROIs. Here all ROIs are defined as nodes. There are also connections between these ROIs. These connections are called endpoints. It is possible that ROIs can be expressed anatomically. This means that ROIs correspond to several anatomical parts of the brain, namely the hippocampus, perirhinal cortex, fissure, pons, and the like [31]. Anatomical atlases in which the cortex is separated according to anatomical features are widely used in neuroimage studies.

In this study, we used multiscale functional brain parcels. Brain parcels were created using a method known as the bootstrap analysis of stable clusters called BASC [32]. Scales were selected using a databased method called MSTEPS [33]. There are different scales in this method. In this study, the scale is set to 122. An edge in a network of brain implies a basic connection between two nodes. An edge between a pair of ROIs is usually weighted with the Pearson correlation coefficient (PCC). For this process, time-series of the brain network obtained from rs-fMRI measurements are used. PCC $r_{x y}$ calculated as follows:

$$
r_{x y}=\frac{\sum_{b=1}^{s}\left(x_{b}-x_{m}\right)\left(y_{b}-y_{m}\right)}{\sqrt{\sum_{b=1}^{S}\left(x_{b}-x_{m}\right)^{2}} \sqrt{\sum_{b=1}^{s}\left(y_{b}-y_{m}\right)^{2}}}
$$

Where, $x$ and $y$ is time series, $\mathrm{s}$ is length, $x_{b}$ and $y_{b}$ are the b-th components of time series, also $x_{m}$ and $y_{m}$ are means of the time series $x$ and $y$. In addition, the pre-processed version of the ABIDE 1 data set was used in this study. Information of the pre-processing phases are given in study [34].

\subsection{Recurrent Neural Network}

Artificial neural networks have a variety of implementation fields. Over time, various versions of artificial neural networks have been specialized in analyzing different types of data. For example, CNNs are specialized for the processing of matrix-type data such as images, whereas recurrent neural networks (RNNs) have also been developed for the processing of sequence data [35]. Traditional feed-forward neural networks (FFNNs) take only existing samples to which they are exposed as input. Differently, RNNs apply what they perceive over time as input as well as existing samples.

The input sequence is given as $\left[x_{1}, x_{2}, \ldots, x_{k}\right]$ and $x_{i} \in \mathbb{R}^{d}$ is allowed. Here the value of $\mathrm{k}$ can vary depending on the length of the sequences. For each step of the RNN model, it generates a hidden state of the sequence $\left[h_{1}, h_{2}, \ldots, h_{k}\right]$. Where, $h_{t-1}$ is the previous hidden state and $x_{t}$ is the current input, the activation of the hidden state at time t can be written as:

$$
h_{t}=f\left(x_{t}, h_{t-1}\right)
$$

Unlike traditional FFNNs, RNNs have a repeating layer. Through this layer, the state information generated by the FFNN is stored and reapplied to the network along with the input information. In other words, RNNs have a memory that keeps what has been calculated until now [36]. Figure 1 shows the representation of the RNN. 


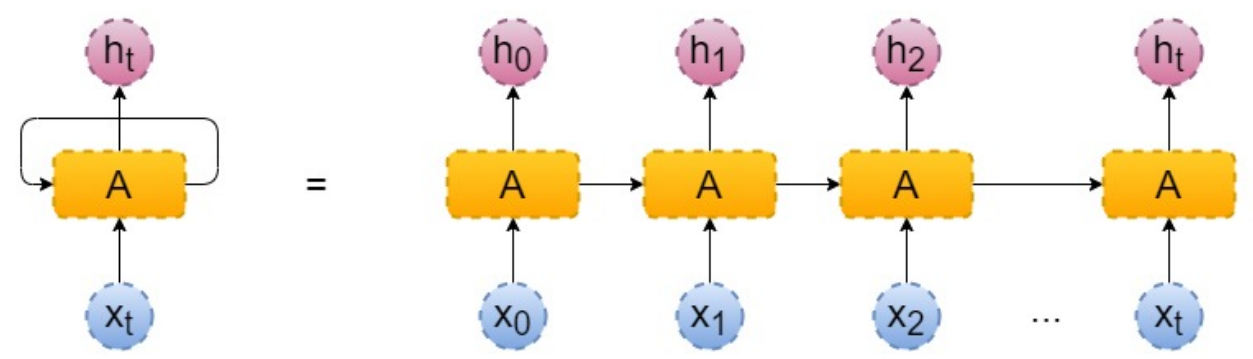

Figure 1 Recurrent neural network representation.

\subsection{Long Short-Term Memory Network}

LSTM is a special form of RNN. It can learn long term dependencies. This model was first proposed in the mid-90s [36] and is widely used today [37]. As mentioned in the above sections, RNNs aim to store and transfer the state information of the artificial neural network while working on arrays. However, it is often not possible to transfer it without breaking long-term dependencies due to the constant processing and transmission of state information.

Although it aims to store and transfer the state information of the artificial neural network while operating on the arrays in RNNs, it is not possible to transfer the status information without breaking the long-term dependencies due to the continuous processing and transfer of the state information. This means the short-term dependencies in the sequence can be effectively transferred. However, there is a problem in the transfer of long-term dependencies. LSTMs are a special model designed to solve this problem.

All RNN-based networks consist of repetitive structures as a chain. In standard RNNs, each of these structures usually consists of a tanh layer or a similar single layer. Figure 2 shows the internal structure of a standard RNN. The feature that distinguishes LSTMs from standard RNNs is that their internal structure is different, as seen in Figure 3.

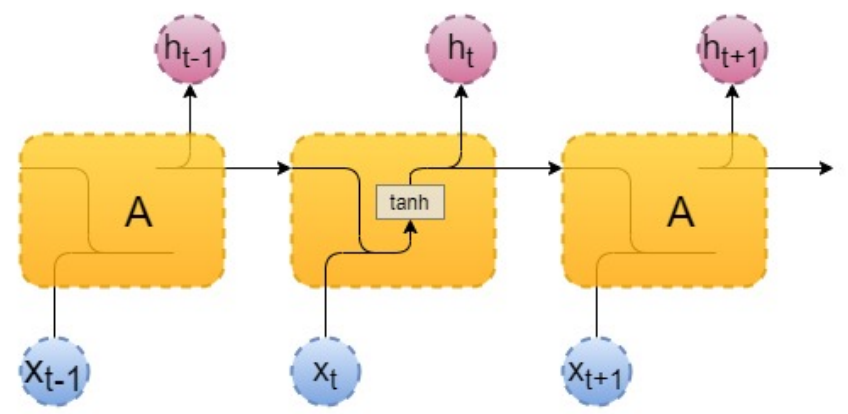

Figure 2 Standard RNN module structure with a single layer.

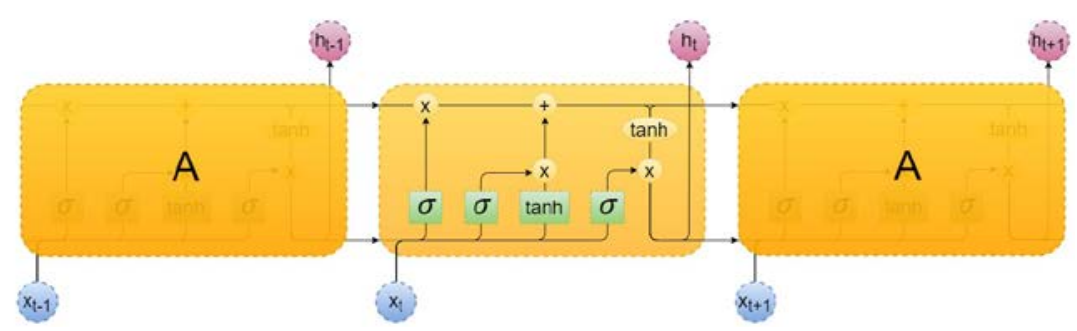

Figure 3 Recurring LSTM module structure with four interactive layers. 
An LSTM module consists of input, forget and output gates. The forget gate uses a sigmoid function. This function takes a value between 0 and 1 . At state 0 , no information is transmitted. 1 means that all must be transmitted. The mathematical model of this gate can be expressed as:

$$
f_{\mathrm{t}}=\sigma\left(\mathrm{W}_{\mathrm{f}}\left[\mathrm{h}_{\mathrm{t}-1}, \mathrm{x}_{\mathrm{t}}\right]+\mathrm{b}_{\mathrm{f}}\right)
$$

Then another sigmoid function decides what information needs to be updated. In addition, a list of candidate values is created and the two operations are combined. Where $\widetilde{C_{t}}$ is a list of candidate values, these two operations can be expressed mathematically as follows:

$$
\begin{gathered}
\mathrm{i}_{\mathrm{t}}=\sigma\left(\mathrm{W}_{\mathrm{i}}\left[\mathrm{h}_{\mathrm{t}-1}, \mathrm{x}_{\mathrm{t}}\right]+\mathrm{b}_{\mathrm{i}}\right) \\
\tilde{\mathrm{C}}=\tanh \left(\mathrm{W}_{\mathrm{C}}\left[\mathrm{h}_{\mathrm{t}-1}, \mathrm{x}_{\mathrm{t}}\right]+\mathrm{b}_{\mathrm{C}}\right)
\end{gathered}
$$

The new status information of the memory cell can be calculated as follows:

$$
\mathrm{C}_{\mathrm{t}}=\mathrm{f}_{\mathrm{t}} \mathrm{C}_{\mathrm{t}-1}+\mathrm{i}_{\mathrm{t}} \tilde{\mathrm{C}}_{\mathrm{t}}
$$

Finally, the output of the system $h_{t}$ is calculated as follows:

$$
\mathrm{h}_{\mathrm{t}}=\mathrm{o}_{\mathrm{t}} \tanh \left(\mathrm{C}_{\mathrm{t}}\right)
$$

\subsection{Bidirectional RNN Models}

In the standard RNN and LSTM models, the representations of previous time steps are learned. In some of its applications, the content may need to be learned in its future representations in order to better grasp the content and remove ambiguities. It is performed in both processes in bidirectional models. The main difference is that the forward propagation process is calculated in two steps. First of all, values are calculated from the first time step to the last time step. Then, starting from the last time step, the values are calculated up to the first time step.

\subsection{Convolutional Neural Networks}

CNN, a type of multiple layered neural network model, was first recommended for computer based vision problems $[35,38]$ and has been used successfully in many image-related applications. CNN's are largely similar to feed-forward neural networks but show differences in the connection between neurons in neighbor layers. CNN models are commonly used by convolution, fully connected and pooling layers.

The size of filter and the generated quantity of maps are used to identify the convolution layer. This layer is the most basic and important unit that forms the CNN. The convolution layer neurons are linked to just a miniature piece of the inlet and extend across the whole measurement of the data applied to the inlet. Then, in the forward propagation phase, dot multiplication is performed between the input data and the filters and a 2-dimension of activation map is created. However, the information required to learn the CNN model is generally nonlinear, but the convolution process is a method involving linear operations such as matrix multiplication and others. Therefore, the non-linearity of the CNN model is improved by applying ReLU (Rectified Linear Units), which is an unsaturated activation function. ReLU can be expressed as:

$$
f(x)=\left\{\begin{array}{lr}
x, & x \geq 0 \\
0, & \text { otherwise }
\end{array}\right.
$$

Also, another important layer is the pooling layer in which down-sampling operations are performed. There are many pooling methods used in the literature, but the two most commonly used methods are maximum pooling and average pooling. In this study, maximum pooling was used. With this method, the input split into non-overlapping rectangles, and only the maximum value is taken from each subpart. The neurons belonging to the fully connected layer are connected to every neuron in the layers before and after the layer, as in traditional artificial neural networks, and may use in the output layer with softmax or another classifier. 


\section{Model Setup \& Evaluation Metrics}

\subsection{Model Parameters}

In order to compare our study with existing studies, 871 samples in the ABIDE I data set were used [2325 , 39]. While 403 records are associated with patients diagnosed with ASD, the remaining 468 samples constitute the control group.

In this study, 7 different models including standard RNN, LSTM, CNN and these were used for ASD detection. A total of 7381 features were obtained as described in the sections above. Features were applied by adjusting to $61 \times 121$ dimensions in all models.

The proposed CNN model consists of 4 learnable layers. The first layer contains a convolution layer of 32 filters of 3x3 dimensions. In this layer, ReLU is used as the activation function. Next is a dropout layer with a value of 0.2. After the dropout layer, there is a $2 \times 2$ max-pooling layer. After these layers, there are convolution, dropout, and max-pooling layers, respectively. All these layers have the same property values as the first layers. This layer's output is implemented as an entry into a fully connected layer consisting of 150 nodes. The activation function of the fully connected layer is sigmoid. After this stage, there is another dropout layer, which is the value of 0.2 . At the last stage, there is a fully connected layer consisting of 1 node and having a sigmoid activation function.

In the CNN-RNN model, unlike the CNN model given above, instead of a fully connected layer consisting of 150 nodes, there is an LSTM layer consisting of 150 nodes to perceive temporal dependencies. All other values are the same as for the CNN model. The CNN-BiRNN model also has a BiRNN layer instead of the RNN layer.

The first layers of the RNN and LSTM models are respectively RNN and LSTM layers, consisting of 150 units. The activation function of the RNN and LSTM layers is the Scaled Exponential Linear Unit (SELU). With $\lambda$ and $\alpha$ constant values, the SELU function can be expressed as:

$$
\operatorname{selu}(x)=\lambda \begin{cases}x & \text { if } x>0 \\ \alpha e^{x}-\alpha & \text { if } x \leq 0\end{cases}
$$

After this layer, there is a dropout layer of 0.2. The last layer contains a fully connected layer consisting of 1 node. Detailed parameters of models are given in Table 1.

\subsection{Performance Evaluation Metrics}

The rs-fMRI images used in our study consist of two separate classes; these are the patients diagnosed with ASD and the control group. Therefore, a binary classification process is carried out. Several different metrics were used to evaluate the performance of models. The performance evaluation criteria used in this study 10-fold cross-validation, classification accuracy (ACC), sensitivity (SENS) and specificity (SPEC) analysis.

Tests on the ABIDE I data set were carried out using the 10-fold cross-validation method. The main purpose of this process is to use each data for both training and testing.

The formulas used to calculate the classification accuracy can be expressed as follows, where $n$ is the test data set, "cn" is the class of $n$ value, Estimate (n) $n$ is the result of the classification process, and $k$ is the total number of groups in the data set.

$$
\begin{aligned}
& \operatorname{Accuracy}(N)=\frac{\sum_{i=1}^{|N|} \text { estimate }\left(n_{i}\right)}{|N|}, n_{i} \in N \\
& \operatorname{Estimate}(n)= \begin{cases}1, & \text { if estimate }(n)=c n \\
0, & \text { otherwise }\end{cases}
\end{aligned}
$$




$$
\text { Classification Accuracy }(M L)=\frac{\sum_{i=1}^{|k|} \operatorname{Accuracy}\left(N_{i}\right)}{|k|}
$$

ACC alone may not provide enough information to perform the performance evaluation of the classification process.

Therefore, additional evaluations, such as SENS and SPEC, can help evaluate the final performance. Here, the SENS term gives information about the proportion of correctly predicted positive classes, while the SPEC term provides information about the correctly predicted negative classes.

Here, true positive (TP), ASD decision should be made while ASD diagnosis should be made, true negative (TN), control decision should be true negative (TN), false positive (FP), ASD decision when control sample should be, and false negative (FN) SENS and SPEC values can be expressed as follows, including those who were mistakenly decided to control while ASD should be diagnosed. Sensitivity and specificity can be expressed as follows:

$$
\begin{aligned}
& \text { Sensitivity }=\frac{T P}{T P+F N} \\
& \text { Specificity }=\frac{T N}{T N+F P}
\end{aligned}
$$

\begin{tabular}{|c|c|c|c|c|}
\hline & CNN & CNN-RNN & RNN & LSTM \\
\hline 1. Layer & $\begin{array}{l}\text { Convolutional Layer } \\
\text { Filter Count: 32, Filter } \\
\text { Size: 3x3 } \\
\text { Activation: ReLU }\end{array}$ & $\begin{array}{l}\text { Convolutional Layer } \\
\text { Filter Count: 32, Filter } \\
\text { Size: 3x3 } \\
\text { Activation: ReLU }\end{array}$ & $\begin{array}{l}\text { RNN Layer } \\
\text { Node Count: } 150 \\
\text { Activation: SELU }\end{array}$ & $\begin{array}{l}\text { LSTM Layer } \\
\text { Node Count: } 150 \\
\text { Activation: SELU }\end{array}$ \\
\hline 2. Layer & $\begin{array}{l}\text { Dropout Layer } \\
\text { Value:0.2 }\end{array}$ & $\begin{array}{l}\text { Dropout Layer } \\
\text { Value:0.2 }\end{array}$ & $\begin{array}{l}\text { Dropout Layer } \\
\text { Value:0.2 }\end{array}$ & $\begin{array}{l}\text { Dropout Layer } \\
\text { Value:0.2 }\end{array}$ \\
\hline 3. Layer & $\begin{array}{l}\text { Max-pooling } \\
\text { Size: 2x2 }\end{array}$ & $\begin{array}{l}\text { Max-pooling } \\
\text { Size: 2x2 }\end{array}$ & $\begin{array}{l}\text { Fully Connected Layer } \\
\text { Node Count: } 1 \\
\text { Activation: Sigmoid }\end{array}$ & $\begin{array}{l}\text { Fully Connected Layer } \\
\text { Node Count: } 1 \\
\text { Activation: Sigmoid } \\
\end{array}$ \\
\hline 4. Layer & $\begin{array}{l}\text { Convolutional Layer } \\
\text { Filter Count: 32, Filter } \\
\text { Size: 3x3 } \\
\text { Activation: ReLU }\end{array}$ & $\begin{array}{l}\text { Convolutional Layer } \\
\text { Filter Count: 32, Filter } \\
\text { Size: 3x3 } \\
\text { Activation: ReLU }\end{array}$ & & \\
\hline 5. Layer & $\begin{array}{l}\text { Dropout Layer } \\
\text { Value:0.2 }\end{array}$ & $\begin{array}{l}\text { Dropout Layer } \\
\text { Value:0.2 }\end{array}$ & & \\
\hline 6. Layer & $\begin{array}{l}\text { Max-pooling Layer } \\
\text { Size: 2x2 }\end{array}$ & $\begin{array}{l}\text { Max-pooling Layer } \\
\text { Size: 2x2 }\end{array}$ & & \\
\hline 7. Layer & $\begin{array}{l}\text { Fully Connected Layer } \\
\text { Node Count: } 150 \\
\text { Activation: Sigmoid }\end{array}$ & $\begin{array}{l}\text { RNN Layer } \\
\text { Node Count: } 150 \\
\text { Activation: SELU }\end{array}$ & & \\
\hline 8. Layer & $\begin{array}{l}\text { Dropout Layer } \\
\text { Value: } 0.2\end{array}$ & $\begin{array}{l}\text { Dropout Layer } \\
\text { Value:0.2 }\end{array}$ & & \\
\hline 9. Layer & $\begin{array}{l}\text { Fully Connected Layer } \\
\text { Node Count: } 1 \\
\text { Activation: Sigmoid }\end{array}$ & $\begin{array}{l}\text { Fully Connected Layer } \\
\text { Node Count: } 1 \\
\text { Activation: Sigmoid }\end{array}$ & & \\
\hline
\end{tabular}

Table 1 Detailed scanning parameters of models 
Bayram et al.

\section{Results and Discussion}

Early diagnosis of ASD is very important in keeping the progression of the disease under control. On the other hand, today's diagnostic methods are based on observing behavioral disorders caused by the disease. At this stage, the disease has already caused permanent, irreversible damage. In this respect, it is very important to diagnose ASD at an early stage using medical imaging techniques instead of following behavioral disorders [12,13]. In this study, a study on ASD detection on rs-fMRI data is presented. Compared to many techniques such as rs-fMRI, MEG, PET and EEG, it provides much better spatial resolution and an acceptable temporal resolution.

Table 2 Average classification performances

\begin{tabular}{|l|c|c|c|}
\hline METHOD & ACC & SENS & SPEC \\
\hline RNN & $\mathbf{7 4 . 7 4}$ & $\mathbf{7 2 . 9 5}$ & 76.28 \\
\hline BiRNN & 74.51 & 68.73 & 79.49 \\
\hline LSTM & 74.4 & 66.25 & $\mathbf{8 1 . 4 1}$ \\
\hline BiLSTM & 74.51 & 72.21 & 76.5 \\
\hline CNN & 70.26 & 66.01 & 73.93 \\
\hline CNN-RNN & 67.28 & 57.57 & 75.64 \\
\hline CNN-BiRNN & 67.51 & 65.51 & 69.23 \\
\hline
\end{tabular}

Table 2 shows the average classification performances obtained by 10 -fold cross-validation tests. When the classification results are examined, it is seen that the highest performance value is provided by RNN with $74.74 \%$. Similarly, the best score with $72.95 \%$ in the SENS value was again provided by RNN. On the other hand, in SPEC value, the best score belongs to LSTM model. The SPEC value for LSTM was 81.41\%. In general, it is seen that the classification performance of RNN and LSTM based models are very close to each other. On the other hand, the performance of CNN-based models is significantly lower than other models. The classification success for CNN, CNN-RNN and CNN-BiRNN models were $70.26 \%, 67.28 \%$ and $67.51 \%$, respectively. While CNNs are very good at capturing spatial dependencies, RNN-based networks are good at capturing temporal dependencies [30]. Depending on the brain network used here, it can be evaluated that temporal dependencies are significantly more dominant. On the other hand, it is seen that the performances of all LSTM and RNN models are close to each other in terms of classification performance. As mentioned in the above sections, LSTM is essentially a special type of standard RNN model. It is successful in eliminating the disadvantages of standard RNN models in capturing long-term dependencies. When the current results obtained with RNN and LSTM models are evaluated, it can be evaluated that long-term dependencies do not affect the classification performance too much. In addition to these, it is seen that the BiRNN and BiLSTM models do not change their classification performance much. However, it appears to affect SENS and SPEC values.

As seen in Graphic 1 and 2, the classification performance of RNN, BiRNN, LSTM and BiLSTM models are very close to each other. However, the SENS values of RNN and BiLSTM models are significantly better than other models. In Graphic 3, box plot representations of SENS values of 4 different models can be seen. Here, the SENS value will decrease if a person who needs to be diagnosed with the disease is mistakenly called healthy. That is, the decrease in the number of FNs is more critical in terms of preventing permanent body damage that may be caused by the disease at an early stage. Graph 1 shows the comparison of ACC values on a fold basis. Also, in Graph 2, boxplot representation of ACC values obtained from RNN, BiRNN, LSTM and BiLSTM models can be seen. When the results are evaluated, it is seen that the RNN model provides stable results in a narrower range. When the values given in Graph 2 are examined, it is seen that the behaviors of the SENS values of RNN and BiLSTM models are close to each other. However, it is seen that the peak values obtained for SENS in the RNN model are better. As a result, it can be evaluated that long-term dependencies do not have a significant effect on performance for the current features used. 


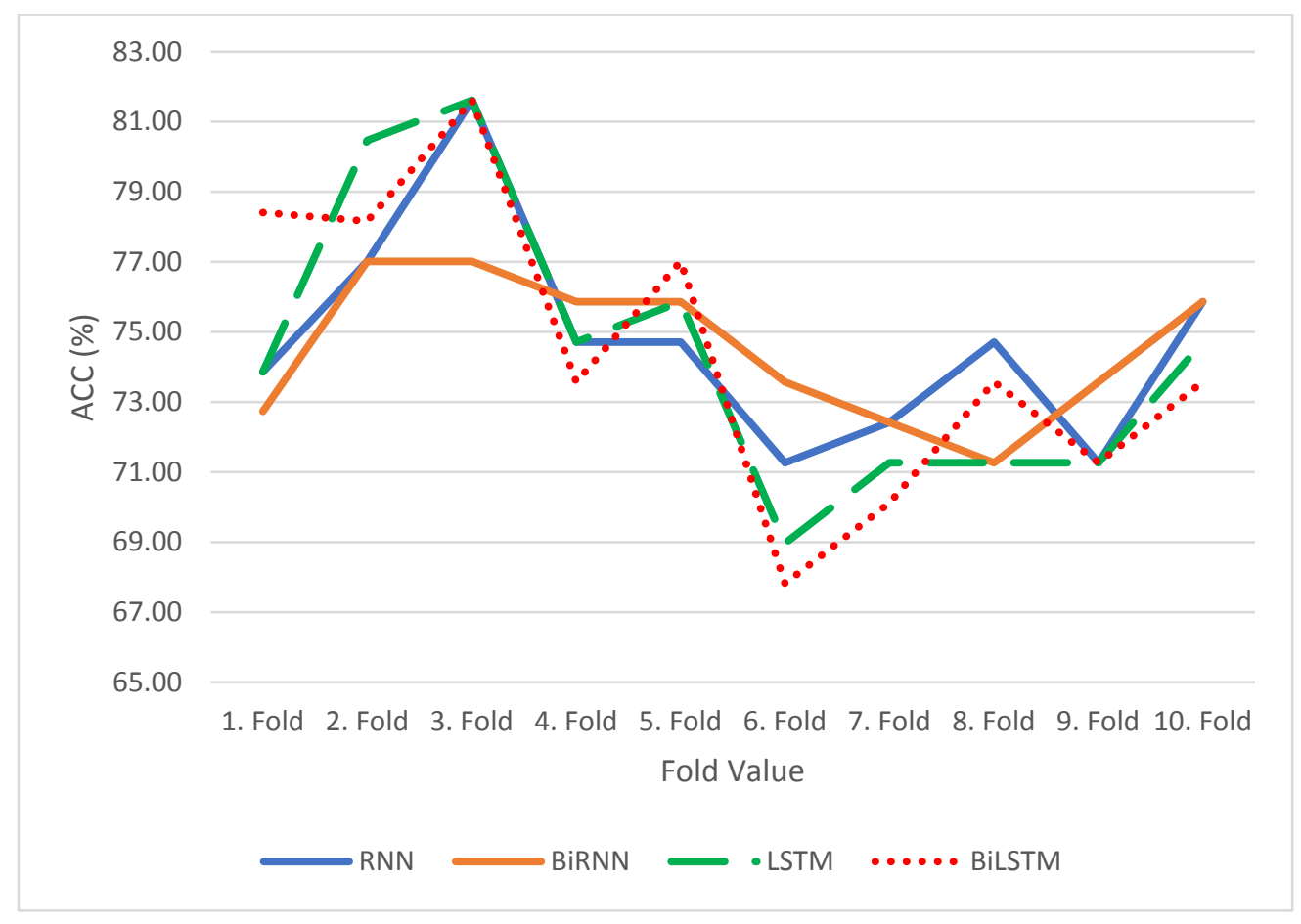

Graph 1 Comparison of ACC values on a fold basis

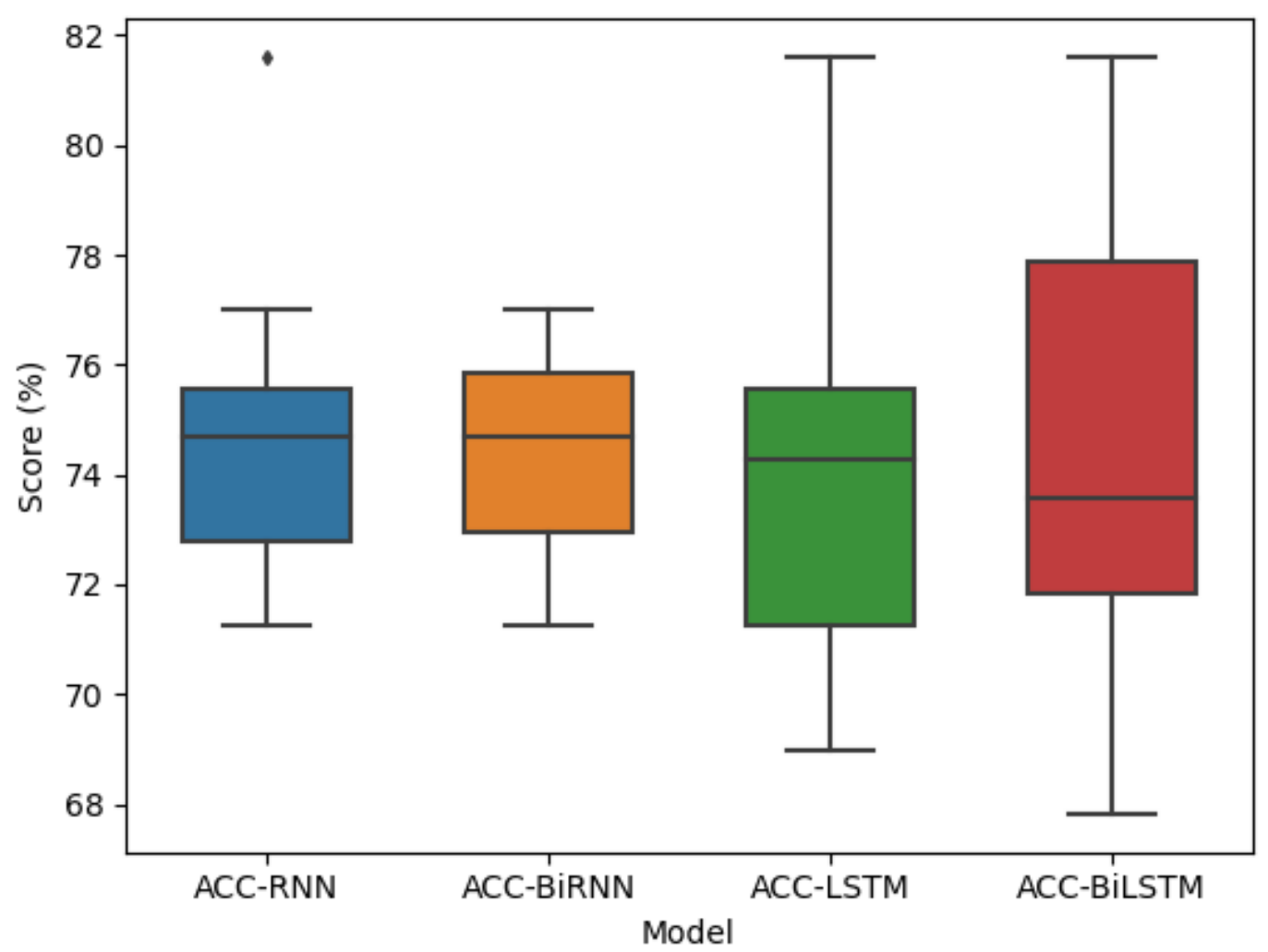

Graph 2 Box plot representation of ACC values on a fold basis 


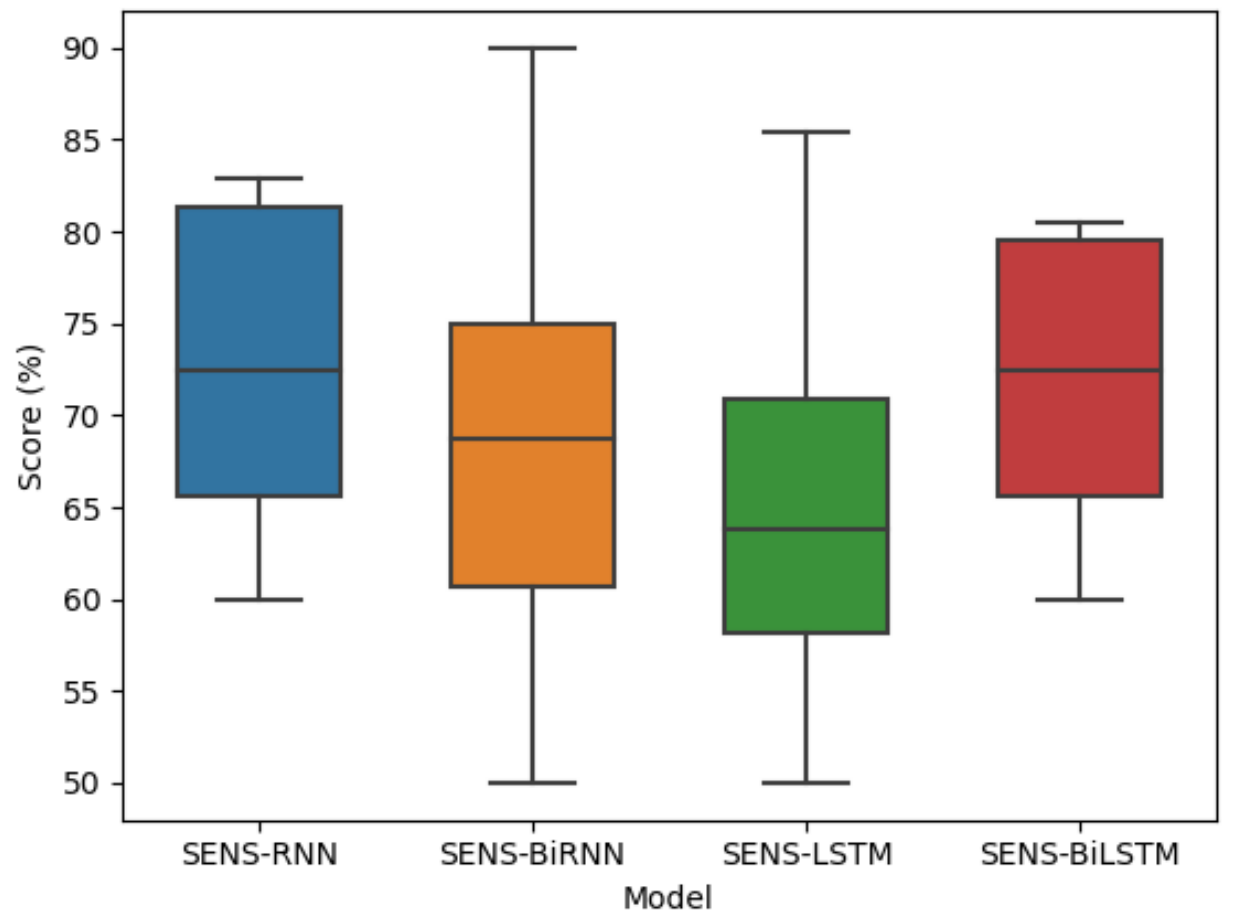

Graph 3 Box plot representation of SENS values on a fold basis

Graph 4 shows a comparison of SPEC values for four models. The highest performance at the average SPEC value belongs to the LSTM model. In the RNN, LSTM, and BiLSTM models, values closer to each other were obtained on the basis of each fold. In the BiRNN model, the lowest and highest values were realized in a wider range than other models. In addition, the SPEC values of all models are higher than SENS values. It is considered that the reason for this is that machine learning models are generally prone to the majority class.

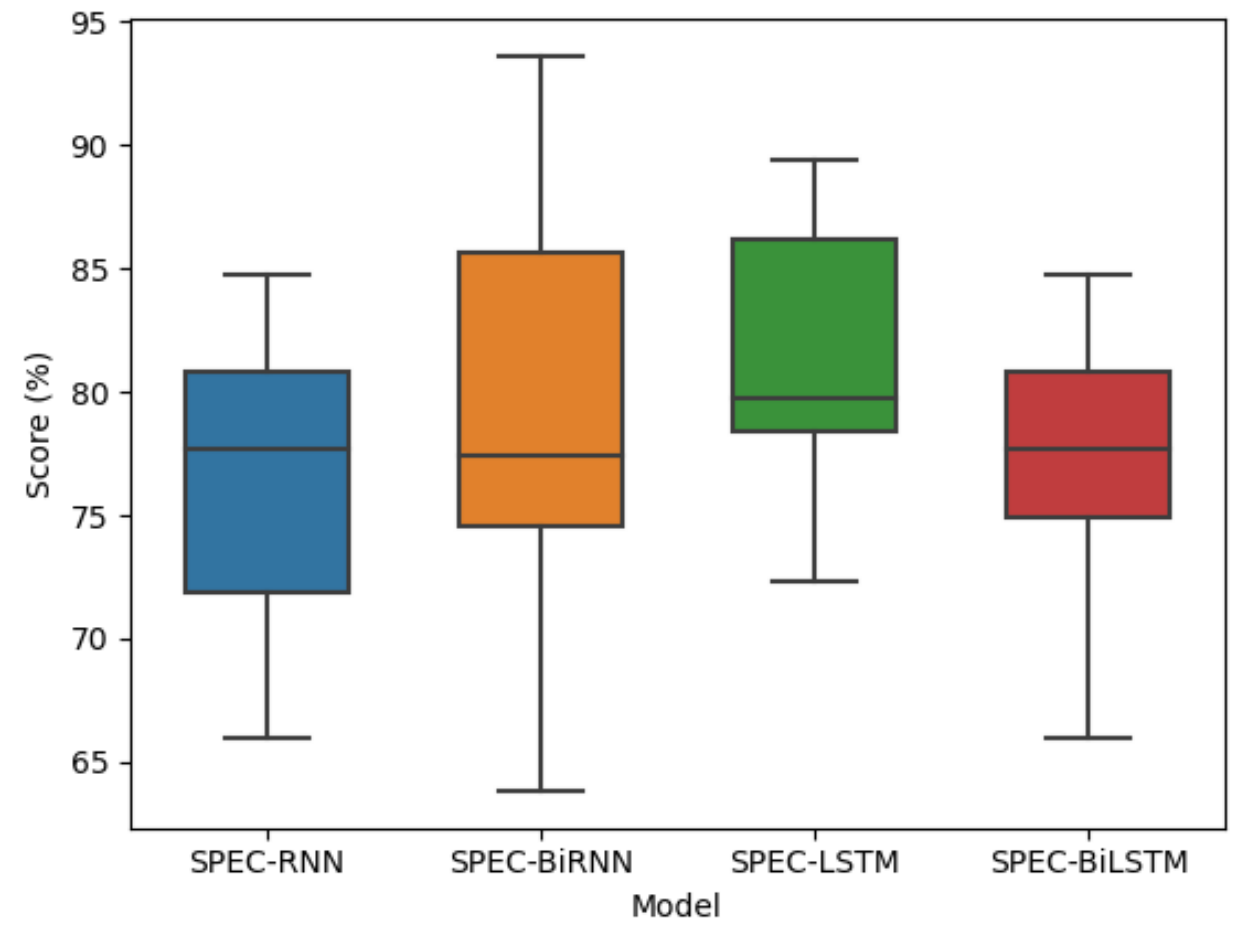

Graph 4 Box plot representation of SPEC values on a fold basis 
Bayram et al.

Finally, we compare our study with other studies in the literature. Table 3 shows the comparison of the models used in this study with other studies in the literature. There are a total of 1112 samples in the ABIDE 1 data set. However, instead of using the entire data set, studies are carried out using different numbers of samples in line with certain criteria. In addition, the properties and classification methods used may differ. In order to make a healthy comparison, we list the sections mentioned above in Table 3. In addition to these, different validation methods can be used in ASD studies. All of the studies shown in Table 3 used 10-fold cross-validation. When the results given in Table 3 are evaluated, it is seen that the highest performance value was obtained in the study performed using power atlas and linear discriminant analysis (LDA) [39]. In the proposed method, a power brain atlas was applied to the preprocessed data. Then, correlation matrices were created using PCC. The feature selection process was applied to the 264x264 dimensional representations created and finally classified with LDA. Here, the performance value was obtained as 77.7\% [39]. The lowest performance value given in Table 3 is Abraham et al. It was obtained in the study performed by [24]. They used more than one brain atlas for ASD detection [24]. As a result, they reached a performance value of $66.8 \%$ in the study [24]. In this study, the second-highest value among the results given in Table 3 was obtained by using a total of 7381 features in 61x121 dimensions. These results confirm the success of RNN-based networks in capturing temporal correlations. It is better than this study with the value obtained in [39]. However, it is evaluated here that the main factor affecting the performance is the extracted features rather than the classification algorithm. In future studies, it is planned to test the models used in this study on different features.

Table 3: Comparison of ACC values with other studies in the literature

\begin{tabular}{|c|c|c|c|c|}
\hline Authors & Features & $\begin{array}{l}\text { Classification } \\
\text { Method }\end{array}$ & $\begin{array}{l}\text { Subject } \\
\text { Number }\end{array}$ & Acc. (\%) \\
\hline Wong et al. [23] & $\begin{array}{c}\text { Harvard-Oxford Atlas / } \\
\text { Riemannian and Correlation Matrix }\end{array}$ & Logistic Regression- $l_{2}$ & 871 & 71.7 \\
\hline Dvornek et al. [40] & Craddock 200 Atlas / Phenotypic Data & LSTM & 1100 & 70.1 \\
\hline $\begin{array}{l}\text { Heinsfeld et al. } \\
\text { [41] }\end{array}$ & Craddock 200 Atlas / PCC & $\begin{array}{c}\text { Denoising } \\
\text { Autoencoder, } \\
\text { Deep Neural Network }\end{array}$ & 1035 & 70 \\
\hline $\begin{array}{l}\text { Abraham et al. } \\
\text { [24] }\end{array}$ & $\begin{array}{l}\text { Multiple Atlas (Harvard-Oxford, } \\
\text { Structural, Yeo Functional, Craddock } \\
200 \text { etc.) }\end{array}$ & $\begin{array}{l}\text { Support Vector } \\
\text { Classifier- } l_{2}\end{array}$ & 871 & 66.8 \\
\hline $\begin{array}{l}\text { Khosla et al. } \\
\text { [43] }\end{array}$ & $\begin{array}{c}\text { Multiple Atlas } \\
\text { (Harvard-Oxford, Craddock 200, } \\
\text { Craddock 400, Dosenbach 160, } \\
\text { Eickhoff-Zilles etc.) } \\
\end{array}$ & 3D CNN & 774 & 73.3 \\
\hline Parisot et al. [25] & Harvard-Oxford Atlas / PCC & $\mathrm{CNN}$ & 871 & 69.5 \\
\hline Mostafa et al. [39] & $\begin{array}{l}\text { Power Atlas / PCC, Sequential Feature } \\
\text { Selection }\end{array}$ & LDA & 871 & 77.7 \\
\hline In this study & BASC Atlas, MSTEPS & Standart RNN & 871 & 74.7 \\
\hline In this study & BASC Atlas, MSTEPS & BiRNN & 871 & 74.5 \\
\hline In this study & BASC Atlas, MSTEPS & LSTM & 871 & 74.4 \\
\hline In this study & BASC Atlas, MSTEPS & BiLSTM & 871 & 74.5 \\
\hline
\end{tabular}


Bayram et al.

\section{Conclusion}

In this study, an approach is presented to detect ASD on rs-fMRI data. Within the scope of the study, 871 samples in the ABIDE I data set were used. While 403 of these samples contain ASD, 468 are control samples.

rs-fMRI data provide information about brain functions in temporal and spatial domains. Accordingly, the performances of RNN-based models, which are very successful in detecting temporal relations between data, and CNN models, which are successful in capturing spatial dependencies, have been compared in this study. The highest ACC and SENS values were obtained with the standard RNN model. ACC and SENS values are $74.74 \%$ and $72.95 \%$, respectively. The classification performance of LSTM models is also very similar to the standard RNN model. However, the performance values of the CNN model are lower compared to these models. In the CNN model, the performance value was obtained at $70.26 \%$.

Classification performance largely depends on extracted features as well as ML algorithms. In future studies, using different brain atlases, their effects on classification performance will be evaluated.

\section{Acknowledgments}

This study has supported by the Scientific Research Project (BAP) Coordinatorship of Bandirma Onyedi Eylül University under grant number BAP-21-1003-003.

\section{References}

[1] M. A. Aghdam, A. Sharifi, and M. M. Pedram, "Diagnosis of Autism Spectrum Disorders in Young Children Based on Resting-State Functional Magnetic Resonance Imaging Data Using Convolutional Neural Networks," Journal of Digital Imaging, vol. 32, no. 6, pp. 899-918, 2019.

[2] B. Crosson et al., "Functional Imaging and Related Techniques: An Introduction for Rehabilitation Researchers," Journal of Rehabilitation Research and Development, vol. 47, no. 2, pp. vii-xxxiv, 2010.

[3] S. Sarraf and J. Sun, "Functional Brain Imaging: A Comprehensive Survey," ArXiv Preprint, arXiv:1602.02225, 2016.

[4] Y. Kong, J. Gao, Y. Xu, Y. Pan, J. Wang and J. Liu, "Classification of Autism Spectrum Disorder by Combining Brain Connectivity and Deep Neural Network Classifier," Neurocomputing, vol. 324, pp. 63-68, 2019.

[5] D. G. Amaral, C. M. Schumann, and C. W. Nordahl, "Neuroanatomy of Autism," Trends in Neurosciences, vol. 31, no. 3, pp. 137-145, 2008.

[6] K. C. Turner, L. Frost, D. Linsenbardt, J. R. Mcllroy and R. Müller, "Atypically Diffuse Functional Connectivity Between Caudate Nuclei and Cerebral Cortex in Autism," Behavioral and Brain Functions, vol. 2, no. 1, p. 34, 2006.

[7] S. J. Blumberg, M. D. Bramlett, M. D. Kogan, L. A. Schieve, J. R. Jones and M. C. Lu, "Changes in prevalence of parent-reported autism spectrum disorder in school-aged US children: 2007 to 2011-2012," National Center for Health Statistics, no. 65, pp. 1-11, 2013.

[8] M. Langen, S. Durston, W. G. Staal, S. J.M.C.Palmen and H. V. Engeland, "Caudate Nucleus Is Enlarged in High-Functioning Medication-Naive Subjects with Autism," Biological psychiatry, vol. 62, no. 3, pp. 262-266, 2007.

[9] M. Coleman and C. Gillberg, "The Autisms," OUP USA, 2012.

[10] L. Waterhouse, "Rethinking Autism: Variation and Complexity,” Academic Press, 2013.

[11] E. Fernell, M. A. Eriksson, and C. Gillberg, "Early Diagnosis of Autism and Impact on Prognosis: a Narrative Review," Clinical Epidemiology, vol. 5, pp. 33-43, 2013.

[12] B. E. Yerys and B. F. Pennington, "How do we establish a biological marker for a behaviorally defined disorder? Autism as a test case," Autism Research, vol. 4, no. 4, pp. 239-241, 2011.

[13] M. Plitt, K. A. Barnes, and A. Martin. "Functional connectivity classification of autism identifies highly predictive brain features but falls short of biomarker standards," NeuroImage: Clinical, 
vol. 7, pp. 359-366, 2015.

[14] C. M. Bishop, "Pattern Recognition and Machine Learning," Springer-Verlag New York Inc., Secaucus, NJ, USA, 2006.

[15] A. L. Samuel, "Some Studies in Machine Learning Using the Game of Checkers," IBM J. Res. Dev., vol. 3, no. 3, pp. 210-229, Jul. 1959.

[16] T. Hastie, R. Tibshirani, and J. Friedman, “The Elements of Statistical Learning: Data Mining, Inference, and Prediction," Springer Series in Statistic, 2009.

[17] M. Uçar and E. Uçar, "Computer-aided detection of lung nodules in chest X-rays using deep convolutional neural networks," Sakarya University Journal of Computer and Information Sciences, vol. 2, no. 1, pp. 1-8, 2019.

[18] Y. Alakoç, V. Akdoğan, M. Korkmaz and O. Er, "Pre-Diagnosis of Osteoporosis Using Probabilistic Neural Networks," Sakarya University Journal of Computer and Information Sciences, vol. 1, no. 3, pp. 1-6, 2018.

[19] E. Erdem and T. Aydin, "Detection of Pneumonia with a Novel CNN-based Approach," Sakarya University Journal of Computer and Information Sciences, vol. 4, no. 1, pp. 26-34, 2021.

[20] D. B. Aydın and O. Er, "A new proposal for early stage diagnosis of urinary tract infection using computers aid systems," Sakarya University Journal of Computer and Information Sciences, vol. 1, no. 1, pp. 1-9, 2018.

[21] G. Ozen, R. Sultanov, Y. Özen and Z. Y. Güneş, "A Convolutional Neural Network Based on Raw Single Channel EEG for Automatic Sleep Staging," Sakarya University Journal of Computer and Information Sciences, vol. 3, no. 2, pp. 149-158, 2020.

[22] A. Di Martino et al., "The Autism Brain Imaging Data Exchange: towards a large-scale evaluation of the intrinsic brain architecture in autism," Molecular Psychiatry, vol. 19, no. 6, pp. 659-667, 2014.

[23] E. Wong, J. S. Anderson, B. A. Zielinski and P. T. Fletcher, "Riemannian regression and classification models of brain networks applied to autism," International Workshop on Connectomics in Neuroimaging, Springer, Cham, 2018.

[24] A. Abraham, M. Milham, A. D. Martino, R. C. Craddock, D. Samaras, B. Thirion and G. Varoquaux, "Deriving reproducible biomarkers from multi-site resting-state data: An Autismbased example," NeuroImage, vol. 147, pp. 736-745, 2017.

[25] S. Parisot, S. I. Ktena, E. Ferrante, M. Lee, R. G. Moreno, B. Glocker and D. Rueckert, "Spectral graph convolutions for population-based disease prediction," International conference on medical image computing and computer-assisted intervention, Springer, Cham, 2017.

[26] J. Wang et al., "Parcellation-dependent small-world brain functional networks: A resting-state fMRI study," Human brain mapping, vol. 30, no. 5, pp. 1511-1523, 2009.

[27] A. Zalesky, A. Fornito, I. H. Harding, L. Cocchi, M. Yücel, C. Pantelis and E. T. Bullmore, "Whole-brain anatomical networks: does the choice of nodes matter?," Neuroimage, vol. 50, no. 3, pp. 970-983, 2010.

[28] C. T. Butts, "Revisiting the foundations of network analysis, " Science, vol. 325, no. 5939, pp. 414-416, 2009.

[29] M. Jenkinson, P. Bannister, M. Brady and S. Smith, "Improved optimization for the robust and accurate linear registration and motion correction of brain images," Neuroimage, vol. 17, no. 2, pp. 825-841, 2002.

[30] Z. Long, X. Duan, D. Mantini, and H. Chen, "Alteration of functional connectivity in autism spectrum disorder: effect of age and anatomical distance," Scientific Reports, vol. 6, no. 1, pp. 18, 2016.

[31] P. Fransson, U. Aden, M. Blennow and H. Lagercrantz, "The functional architecture of the infant brain as revealed by resting-state fMRI," Cerebral Cortex, vol. 21, no. 1, pp. 145-154, 2011.

[32] P. Bellec, P. Rosa-Neto, O. C. Lyttelton, H. Benali, A. C. Evans, "Multi-level bootstrap analysis of stable clusters in resting-state fMRI," Neuroimage, vol. 51, no. 3, pp. 1126-1139, 2010.

[33] P. Bellec, "Mining the hierarchy of resting-state brain networks: selection of representative clusters in a multiscale structure," International Workshop on Pattern Recognition in Neuroimaging, IEEE, 2013.

[34] C. Craddock et al., "The neuro bureau preprocessing initiative: open sharing of preprocessed 
neuroimaging data and derivatives," Frontiers in Neuroinformatics, 2013.

[35] A. T. Kabakuş, "A Comparison of the State-of-the-Art Deep Learning Platforms: An Experimental Study," Sakarya University Journal of Computer and Information Sciences, vol. 3, no. 3, pp. 169182, 2020.

[36] Z. Özer, "The Effect of Normalization on the Classification of Traffic Comments," Ph. D. Thesis, Karabük Unv. Grad. Sch. of Nat. and App. Sci, Dept. of Computer Engineering, Karabük, Turkey, 2019.

[37] A. A. Müngen, İ. Aygün, and M. Kaya, "News and Social Media Users Emotions in the COVID19 Process," Sakarya University Journal of Computer and Information Sciences, vol. 3, no. 3, pp. 250-263, 2020.

[38] Y. LeCun, L. Bottou, Y. Bengio and P. Haffner "Gradient-based learning applied to document recognition," Proceedings of the IEEE, vol. 86, no. 11, pp. 2278-2324, 1998.

[39] S. Mostafa, L. Tang, and F-X. Wu, "Diagnosis of Autism Spectrum Disorder Based on Eigenvalues of Brain Networks," IEEE Access, vol. 7, pp. 128474-128486, 2019.

[40] N. C. Dvornek, P. Ventola, and J. S. Duncan, "Combining phenotypic and resting-state fMRI data for autism classification with recurrent neural networks," IEEE 2018 15th International Symposium on Biomedical Imaging, IEEE, 2018.

[41] A. S. Heinsfeld, A. R. Franco, R. C. Craddock, A. Buchweitz and F. Meneguzzi "Identification of autism spectrum disorder using deep learning and the ABIDE dataset," NeuroImage: Clinical, vol. 17, pp. 16-23, 2017.

[42] H. Sharif and R. A. Khan, "A novel framework for automatic detection of autism: A study on corpus callosum and intracranial brain volume," arXiv preprint, arXiv:1903.11323, 2019.

[43] M. Khosla, K. Jamison, A. Kuceyeski, and M. R. Sabuncu, "3D convolutional neural networks for classification of functional connectomes," Deep Learning in Medical Image Analysis and Multimodal Learning for Clinical Decision Support, Springer, Cham, pp. 137-145, 2018. 